\title{
NMR Shift and Relaxation and the Electronic Spin of Superconducting Cuprates
}

\author{
Marija Avramovska ${ }^{1}$. Danica Pavićević ${ }^{1}$ Jürgen Haase ${ }^{1}$
}

Received: 24 March 2020 / Accepted: 28 March 2020 / Published online: 25 April 2020

(C) The Author(s) 2020

\begin{abstract}
Very recently, by inspecting large sets of data across all families of superconducting cuprates, it became obvious that the prevailing nuclear magnetic resonance (NMR) interpretation of cuprate properties is not adequate, as it does not account for the differences between the families, as well as common characteristics beyond simple temperature dependence. From the most abundant planar $\mathrm{Cu}$ shift data, one concludes readily on two electronic spin components with different doping and temperature dependencies. Their uniform response that causes NMR spin shifts consists of a doping-dependent component due to planar $\mathrm{O}$, and another due to spin in the planar copper $3 d\left(x^{2}-y^{2}\right)$ orbital, where the latter points opposite the field direction. Planar $\mathrm{Cu}$ relaxation was found to be rather ubiquitous (except for $\mathrm{La}_{2-x} \mathrm{Sr}_{x} \mathrm{CuO}_{4}$ ), and Fermi liquid-like, i.e., independent of doping and material, apart from the sudden drop at the superconducting transition temperature, $T_{\mathrm{c}}$. Only the relaxation anisotropy is doping and material dependent. We showed previously that one can understand the shifts within a two-component scenario, but we failed with a model to account for the relaxation. Here, we suggest a slightly different shift scenario, still based on the two components, by introducing different hyperfine couplings, and, importantly, we are able to account for the $\mathrm{Cu}$ nuclear relaxation and its anisotropy for all materials, including also $\mathrm{La}_{2-x} \mathrm{Sr}_{x} \mathrm{CuO}_{4}$. The results represent a solid framework for theory.
\end{abstract}

Keywords Cuprates $\cdot$ NMR $\cdot$ Electronic properties

\section{Introduction}

Nuclear magnetic resonance (NMR) is a powerful local, bulk probe of material properties [1]. This concerns the chemical as well as electronic structure of materials, which can be studied locally at various nuclear sites in the unit cell. The changes in the NMR shifts and relaxation from the modification of the density of states due to the opening of a superconducting gap in conventional superconductors are famous examples [2,3]. Not surprisingly, after the discovery of cuprate superconductivity [4], NMR experiments focused

Marija Avramovska

marija.avramovska@uni-leipzig.de

Danica Pavićević

danicas.dp@gmail.com

Jürgen Haase

j.haase@physik.uni-leipzig.de

1 Felix Bloch Institute for Solid State Physics, University of Leipzig, Linnéstr. 5, 04103, Leipzig, Germany in particular on planar $\mathrm{Cu}$ and $\mathrm{O}$ in these type II materials (for reviews of cuprate NMR, see [5, 6]). However, with the early focus on only a few systems and a lack of established theory, the NMR data interpretation ceased to evolve with a number of questions unanswered. Fortunately, more and more NMR studies of different materials appeared in the literature over the years.

During the last 10 years, with special NMR experiments on $\mathrm{La}_{1.85} \mathrm{~S}_{0.15} \mathrm{CuO}_{4}$ [7], $\mathrm{YBa}_{2} \mathrm{Cu}_{4} \mathrm{O}_{8}$ [8], and samples of the $\mathrm{HgBa}_{2} \mathrm{CuO}_{4+\delta}$ family of materials $[9,10]$, a cornerstone of the old interpretation was questioned and shown to be not correct: a single temperature-dependent electronic spin component, $s(T)$, that follows from the uniform spin susceptibility, i.e., $s(T)=\chi(T) \cdot B_{0}$, in an external field $B_{0}$, is not capable of describing the temperature-dependent NMR spin shifts, ${ }^{n} K_{d}(T)={ }^{n} H_{d} \cdot \chi(T)$. Since ${ }^{n} K_{d}(T)$ can be measured at various nuclei $(n)$, or for any orientation (d) of the external field with respect to the crystal axes, with ${ }^{n} H_{d}$ being the corresponding hyperfine constant, one demands from different experiments that $\Delta^{n} K_{d} \propto \Delta^{m} K_{e}$, which was clearly not observed in general, only in certain ranges of temperature [7-10]. 
Also during the last decade, the understanding of the charge sharing in the $\mathrm{CuO}_{2}$ plane advanced significantly from a more qualitative [11] into a quantitative model $[12,27]$. It became apparent that, e.g., the maximum temperature of superconductivity correlates with the sharing of the inherent hole between planar $\mathrm{Cu}$ and $\mathrm{O}$, the higher the oxygen hole content the higher $T_{\mathrm{c}, \max }$ [13]. Other cuprate properties depend on the charge sharing, as well. This mostly family-dependent behavior stimulated some of us to inspect also a larger body of NMR shifts and relaxation for material-dependent differences or common characteristics.

In the first step, all available ${ }^{63} \mathrm{Cu}$ NMR shifts that are rather abundant and reliable were gathered [14]. And, indeed, by just plotting these shifts a new phenomenology emerged, and points immediately to a more complicated uniform response that cannot be explained with a simple $\chi(T)$. In the second step, all available ${ }^{63} \mathrm{Cu}$ NMR relaxation rates were collected $[15,16]$ and, again, simple plots revealed a surprisingly different scenario. Here, a rather material- and doping-independent relaxation was revealed with spin fluctuations similar, but not in excess to what one expects from a simple Fermi liquid. Only the relaxation anisotropy depends on the materials and decreases with increasing doping.

Then, in a first attempt, we tried to reconcile these findings [15]. We could show that a two-component description, as introduced earlier [7] (with two spin components that couple with two different hyperfine coefficients, ${ }^{n} H_{1 d}$ and ${ }^{n} H_{2 d}$, to each nuclear spin, $n$ ), is indeed sufficient to understand the planar $\mathrm{Cu}$ shifts (with the $\mathrm{La}_{2-x} \mathrm{Sr}_{x} \mathrm{CuO}_{4}$ family being some kind of outlier, cf. Fig. 1). Two spin susceptibilities $\left(\chi_{1}, \chi_{2}\right)$ demand a third term from a coupling between the two electronic spin components. That is, one has to write, $\chi_{1}=\chi_{11}+\chi_{12}, \chi_{2}=$ $\chi_{22}+\chi_{21}\left(\chi_{12}=\chi_{21}\right)$, and,

$$
{ }^{n} K_{\|, \perp}={ }^{n} H_{1 \|, \perp} \cdot(a+c)+{ }^{n} H_{2 \|, \perp} \cdot(b+c)
$$

with $a=\chi_{11} B_{0}, b=\chi_{22} B_{0}$, and $c=\chi_{12} B_{0}$, and the magnetic field parallel and perpendicular to the crystal $c$-axis.

In order to independently test the important conclusion of two spin components with different doping and temperature dependencies, we investigated the planar $\mathrm{O}$ data [20], very recently. We found that, indeed, planar $\mathrm{O}$ shifts demand two spin components, as well, where one of them is doping dependent. This encouraged us to search for a better understanding of the very reliable $\mathrm{Cu}$ data, in particular of the nuclear relaxation and its anisotropy, which we failed to deliver previously [15].

By plotting a large set of literature $\mathrm{Cu}$ relaxation data [15], we found generic behavior, as well, with the exception of just one family, $\mathrm{La}_{2-x} \mathrm{Sr}_{x} \mathrm{CuO}_{4}$. In fact, all other cuprates have rather similar relaxation rates, $1 / T_{1 \perp}$ [16], i.e., if measured with the magnetic field perpendicular to the crystal $c$-axis, $c \perp B_{0}$, cf. Fig. 2. In particular, just above $T_{\mathrm{c}}$ the value of $1 / T_{1 \perp} T_{\mathrm{c}} \sim 20 / K s$ for all cuprates, while $T_{\mathrm{c}}$ can be very different, or even close to zero for strongly overdoped systems. There is no particular doping dependence of $1 / T_{1 \perp}$ as one might naively expect if electronic spin fluctuations beyond those of a more regular Fermi liquid were to increase toward lower doping levels (there are hardly data available at very low doping). In fact, a value of $20 / \mathrm{Ks}$ follows with the Korringa relation [22] from those cuprates with the highest shifts, i.e., the upper right corner of the shaded triangle in Fig. 1 [15], which suggests that the shifts have the tendency to be suppressed if the Korringa relation fails, and it is not due to an increased relaxation. The situation is somewhat different for $1 / T_{1 \|}$ since for this direction of measurement $\left(c \| B_{0}\right)$ the rates differ between families and have the tendency to increase with decreasing doping. However, it was demonstrated that the ratio, $\left[1 / T_{1 \perp}(T)\right] /\left[1 / T_{1 \|}(T)\right]$, is temperature independent for all cuprates, and is the same above and below $T_{\mathrm{c}}$; i.e., both rates are proportional to each other $[15,16]$.

Interestingly, the $\mathrm{La}_{2-x} \mathrm{Sr}_{x} \mathrm{CuO}_{4}$ family of materials is the only outlier to this phenomenology, cf. Fig. 2. However, the anisotropy ratio is also temperature independent and has a value of about 2.3 , very similar to that of some other cuprates.

In our first attempt at reconciling shift and relaxation [15], we could only explain the shift suppression, but failed to present a microscopic model that also explains the relaxation and its anisotropy. Here, we discuss and modify our previously suggested two-component scenario [15] in that we introduce somewhat different hyperfine coefficients, together with a new notation, still based on the identification of the two electronic components as being due to planar $\mathrm{Cu} 3 d\left(x^{2}-y^{2}\right)$ and likely planar $\mathrm{O} 2 p_{\sigma}$ spin densities. Most importantly, we are able to present a simple, yet fundamental, model of nuclear relaxation in terms of these two components that fits all planar $\mathrm{Cu}$ relaxation data, even including the outlier $\mathrm{La}_{2-x} \mathrm{Sr}_{x} \mathrm{CuO}_{4}$.

\section{Planar Cu Shifts}

Dissimilar from our previous attempt to understand all planar $\mathrm{Cu}$ shift data [15], we will be using a somewhat different nomenclature and hyperfine coupling coefficients here. Therefore, we repeat the basic arguments leading to the description, now.

The total magnetic shift for planar $\mathrm{Cu},{ }^{63} \hat{K}_{\|, \perp}$, is the sum of an orbital and spin shift component, and we have for 


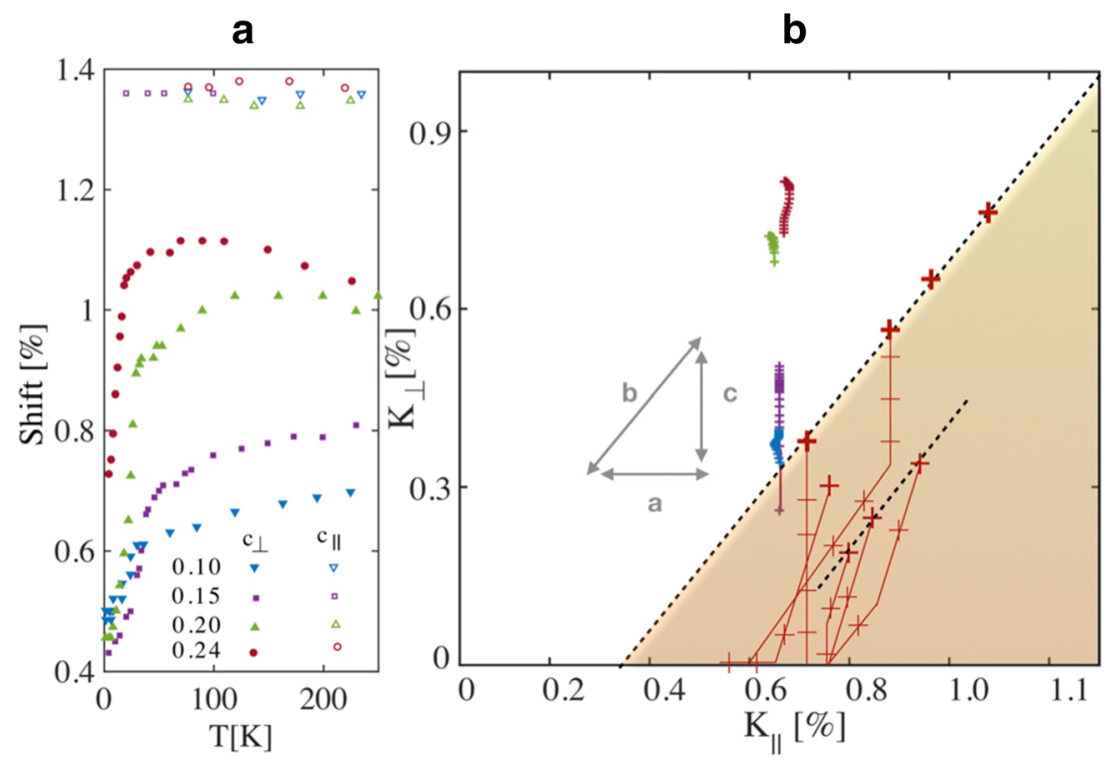

Fig. 1 a Total ${ }^{63} \mathrm{Cu}$ shifts vs. temperature, $\hat{K}_{\|, \perp}(T)$, for 4 doping levels of $\mathrm{La}_{2-x} \mathrm{Sr}_{x} \mathrm{CuO}_{4}$ and two directions $\left(c \| B_{0}, c \perp B_{0}\right)$ of the external field $B_{0}$ with respect to the crystal $c$-axis (adopted from [17]). $K_{\|}$is $T$ independent and similar for all doping levels. $K_{\perp}$ shows a much larger spread with doping and decreases rapidly near $T_{\mathrm{c}}$. b Sketch of the spin shift $K_{\perp}(T)$ vs $K_{\|}(T)$ plot valid for other cuprates [14], with real data from the 4 doping levels of $\mathrm{La}_{2-x} \mathrm{Sr}_{x} \mathrm{CuO}_{4}$ (with temperature as an implicit parameter). The shaded area is where the rest of the many cuprates can be found (the shaded triangle has a hypotenuse of slope $\approx 1$ ), only typical data are shown by crosses. Data

the two orientations $\left(c \| B_{0}, c \perp B_{0}\right)$ of the magnetic field $B_{0}$ with respect to the crystal $c$-axis,

$\hat{K}_{\|, \perp}(T)=K_{L \|, \perp}+K_{\|, \perp}(T)$.

It is of particular use to plot the total shifts $\hat{K}_{\perp}(T)$ vs. $\hat{K}_{\perp}(T)[14]$ with temperature as an implicit parameter, i.e., one does not make assumptions about $K_{L \|, \perp}$. Such a plot brings out a number of remarkable trends $[14,15]$. A sketch of such a plot is presented in Fig. 1b, and we repeat some conclusions [15], but also include new ones, below. lie on straight line segments (lines) with a few slopes only: a slope $\approx 1$ (dashed lines); a very steep slope (vertical lines); a slope of 2.5 . For example, a slope of 2.5 is typical for $\mathrm{HgBa}_{2} \mathrm{CuO}_{4+\delta}$ (at higher $T$ ), a steep slope for $\mathrm{YBa}_{2} \mathrm{Cu}_{4} \mathrm{O}_{8}$, and for symmetry reasons we use a slope of 1 for some Tl-based compounds, as well as for overdoped $\mathrm{HgBa}_{2} \mathrm{CuO}_{4+\delta}$ at low $T$. $\mathrm{La}_{2-x} \mathrm{Sr}_{x} \mathrm{CuO}_{4}$ is a clear outlier with only the steep slope. In the simple two-component description, cf. (5), (6), a change in one of the components $a, b$, or the coupling $c$ leads to the indicated slopes in the middle of (b); for the subtraction of the orbital shifts, see main text

A fundamental assumption is $[14,15]$,

$K_{L \perp} \approx 0.30 \%$,

since all cuprates show a rather similar low temperature shift for $c \perp B_{0}$ with $\hat{K}_{\perp}(T \rightarrow 0) \approx 0.30 \%$. Therefore, this value appears to be reliable. It is the same assumption made early on [5]. We note that this value is backed by first principle calculations [19] (while this is not the case for $K_{L \|}$ ).

Second, except for $\mathrm{La}_{2}{ }_{x} \mathrm{Sr}_{x} \mathrm{CuO}_{4}$, all data points in that plot are found in the lower right triangle that has
Fig. 2 a Planar ${ }^{63} \mathrm{Cu}$ relaxation rates of the cuprates (data from [16]); $1 / T_{1 \perp}$ of $\mathrm{La}_{2-x} \mathrm{Sr}_{x} \mathrm{CuO}_{4}$ in comparison is about twice as high as that of other cuprates. b $1 / T_{1 \perp}$ vs $1 / T_{1 \|}$, which is $\approx 2.3$ for $\mathrm{La}_{2-x} \mathrm{Sr}_{x} \mathrm{CuO}_{4}$

(highlighted), is very similar to what is found for other cuprates (data [16]). It is mostly $1 / T_{1 \|}$ that changes with doping and material, but remains proportional to $1 / T_{1 \perp}$ (at all temperatures) a

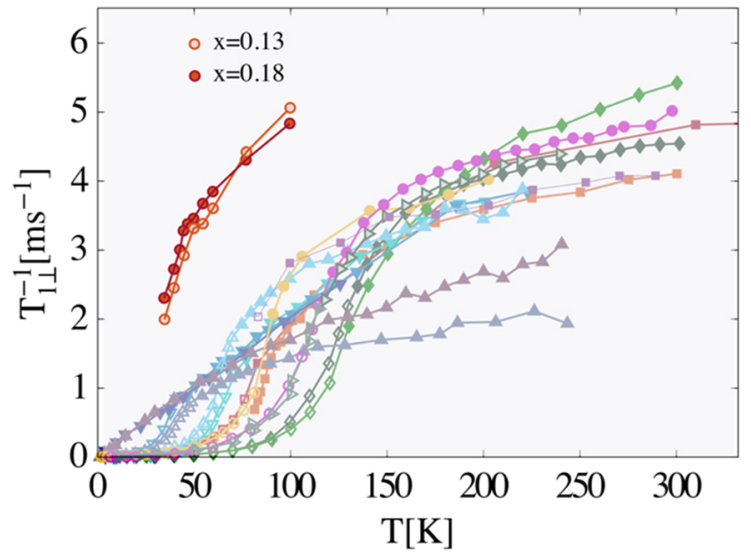

b

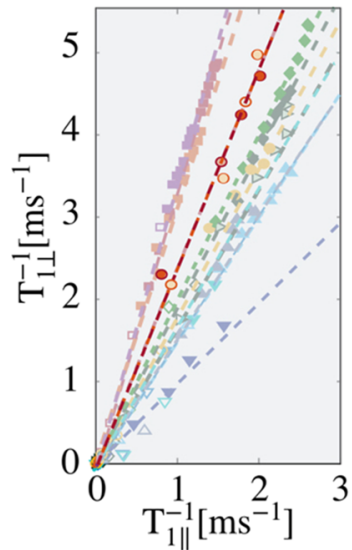


as hypothenuse a line of slope 1 , i.e., $\Delta \hat{K}_{\perp} / \Delta \hat{K}_{\|}(T) \approx$ 1. This line points immediately to an isotropic hyperfine coefficient, while the fact that $\hat{K}_{\|}(T)>\hat{K}_{\perp}(T)$ (all data in the lower, right triangle) demands a second, very anisotropic hyperfine coefficient that acts mostly for $c \| B_{0}$. Very similar arguments as put forward in the old literature let us choose $A_{\perp, \|}$ and $B$ : note that there must be spin in the $3 d\left(x^{2}-y^{2}\right)$ orbital, and it is very likely that there will also be an isotropic coupling term. Then, the NMR shifts demand, however, that the spin polarization in the $3 d\left(x^{2}-y^{2}\right)$ orbital must be negative, as pointed out recently $[14,15]$, for we know that $A_{\|}$is negative, and $\left|A_{\|}\right| \gg A_{\perp}$ [23].

Thus, we write with (1),

$K_{\| \perp}=A_{\|, \perp} \cdot\left(a+4 c_{j}\right)+B \cdot 4\left(b_{j}+c_{j}\right)$.

For symmetry reasons, we take $\left(b_{j}+c_{j}\right)$ from each of the 4 neighbors to be the same, i.e., from spin in the planar O $2 p_{\sigma}$ orbitals, cf. Fig. 3. As before [15], we will neglect $A_{\perp}$ and simply write:

$$
\begin{aligned}
& K_{\|}=A_{\|}\left(a+4 c_{j}\right)+B \cdot 4\left(b_{j}+c_{j}\right) \\
& K_{\perp} \approx B \cdot 4\left(b_{j}+c_{j}\right) .
\end{aligned}
$$

This is a different notation from before [15] where we used $b=4 b_{j}$.

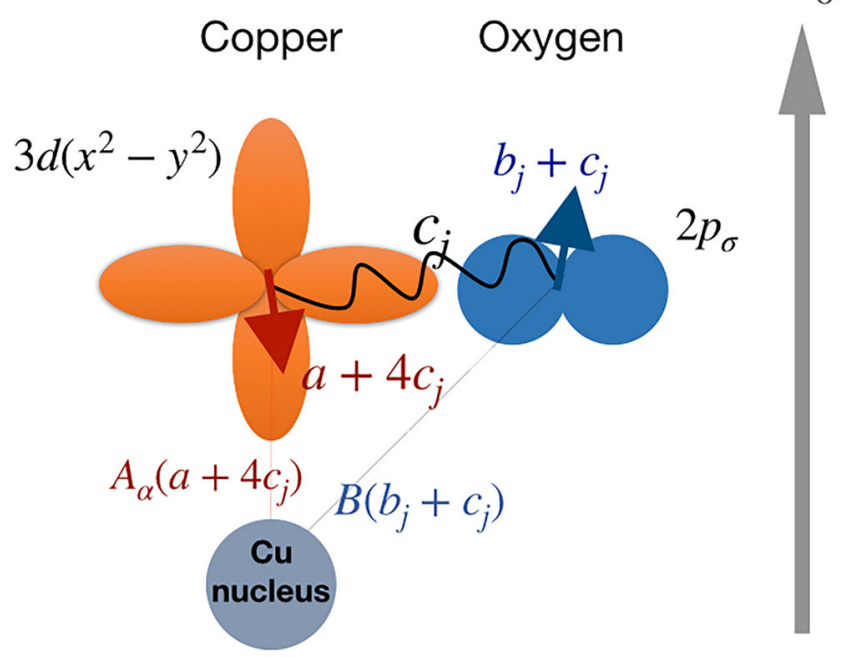

Fig. 3 In an external magnetic field $B_{0}$ two spin components $a$ and $b_{j}$ appear, originating from the planar $\mathrm{Cu} 3 d\left(x^{2}-y^{2}\right)$ and the four surrounding $\mathrm{O} 2 p_{\sigma}$ orbitals, respectively. Due to a coupling $(c)$, the effective components are $\left(a+4 c_{j}\right)$ and $\left(b_{j}+c_{j}\right)$. While $\left(b_{j}+c_{j}\right)$ is positive for the cuprates, $\left(a+4 c_{j}\right)$ turns out to be negative. The hyperfine coefficients $A_{d}$ and $B$ lead to orientation-dependent $(d)$ NMR shifts $K_{d}=A_{d}\left(a+4 c_{j}\right)+B\left(b_{j}+c_{j}\right)$ at the $\mathrm{Cu}$ nucleus
A zero spin shift, our first, fundamental assumption means that $\sum_{j}\left(b_{j}+c_{j}\right)=0$, and we have for the other orientation:

$\hat{K}_{\|}(T \rightarrow 0)=K_{L \|}+A_{\|}\left(a+4 c_{j}\right)$.

In order to estimate the orbital shift, $K_{L \|}$, for this orientation of the field, as before [15], the most reliable approach is to use (3) together with calculations of the orbital shift anisotropy, since the latter is mostly determined by matrix elements involving the orbital bonding wave functions of $\mathrm{Cu}$ and $\mathrm{O}[18,19]$. In fact, we use the suggested value of 2.4 from [19]:

$$
{ }^{63} K_{L \|}=2.4 \cdot{ }^{63} K_{L \perp} \approx 0.72 \% \text {. }
$$

Note that this value could vary between families, but since the orbital shift for $c \perp B_{0}$ does not change significantly between families, we do not expect a large effect for $c \| B_{0}$, as well. This is important as it means that most cuprates have a non-vanishing spin shift for $c \| B_{0}$ from a negative spin polarization in the $3 d\left(x^{2}-y^{2}\right)$ orbital, even at the lowest temperatures.

As mentioned earlier, a few special slopes govern the shift-shift plot presented in their figure 7 [14], and we highlighted them in Fig. 2b, again. These are segments defined by temperature or doping for which the ratio of changes in both shifts is constant, $\Delta \hat{K}_{\perp}(T) / \Delta \hat{K}_{\|}(T)=\kappa$, and one finds 4 slopes, $\kappa \approx 0,1,2.5, \infty$. For example, $\kappa=1$ denotes isotropic shift lines and readily follows from a mere change of $b_{j}$ only, as it enters both terms in (5) and (6). Then, $\kappa \approx 0$ in this approximation is realized by a change in $a$, only, since we neglected the rather small $A_{\perp}$. Note that term $c$ operates on both shifts, $K_{\perp}$ and $K_{\|}$, and must be involved in the special slopes $\kappa=2.5$ and $\kappa \approx \infty$. While not favored before [15], we believe that $\kappa \approx \infty$ is caused by a mere change in $c$. The reasoning is as follows: not a single material in the shift-shift plot shows a negative slope, i.e., a slope to the right of $\kappa \approx \infty$. This is remarkable and must mean that the component $a$ cannot significantly be involved in shift changes.

With this assumption that $c_{j}$ causes $\kappa \approx \infty$, we note that (5) and (6) require:

$A_{\|} \approx-B$,

and we have with (5) and (6):

$$
\begin{aligned}
& K_{\|} \approx B\left(4 b_{j}-a\right) \\
& K_{\perp} \approx B 4\left(b_{j}+c_{j}\right) .
\end{aligned}
$$

Note that in this approximation, $c$ effectively acts only for $c \perp B_{0}$. Then, the slope of $\kappa \approx 2.5$ is given by a concomitant change of $b_{j}$ and $c_{j}$, e.g., $\Delta b_{j}=1.5 \Delta c_{j}$ if both terms change proportionally.

To summarize, in the above model, the individual changes of $a, b_{j}$, and $c_{j}$ correspond to slopes of $\kappa=0,1$, 
and $\infty$, respectively, in Fig. 1 (if all $b_{j}$ and $c_{j}$ are the same). Even if this is not precisely what happens, we think that (10) and (11) still capture the fundamental aspects of the planar $\mathrm{Cu}$ shifts.

With these results in mind, we can look at the data for $\mathrm{La}_{2-x} \mathrm{Sr}_{x} \mathrm{CuO}_{4}$ again.

The high temperature shifts for $\mathrm{La}_{2-x} \mathrm{Sr}_{x} \mathrm{CuO}_{4}, K_{\perp}$, are much larger than what we expect from its $K_{\|}=$ $B\left(4 b_{j}-a\right)$ values. In one scenario, a larger $a$ and larger $b_{j}$ could position this family at larger $K_{\perp}$ (for given $c_{j}$ ). The action of a temperature-dependent $c_{j}$ then leads to the $\kappa \approx \infty$ slope. Alternatively, $c_{j}$ could be much larger for $\mathrm{La}_{2-x} \mathrm{Sr}_{x} \mathrm{CuO}_{4}$, i.e., much more positive, at high temperatures. This also leads to a much larger $B\left(b_{j}+c_{j}\right)$. Again, a drop in $c_{j}$ then makes $\left(b_{j}+c_{j}\right)$ disappear.

To conclude, while $\mathrm{La}_{2-x} \mathrm{Sr}_{x} \mathrm{CuO}_{4}$ is an outlier in the shifts, the position in Fig. 1 can be understood within the two-component scenario, as well.

\section{Planar Cu Relaxation}

The nuclear relaxation rate $1 / T_{1 \|}$ measures the in-plane fluctuating magnetic fields, $\left\langle h_{\perp}^{2}\right\rangle$, from electronic spin fluctuations, while $1 / T_{1 \perp}$ is affected by both, in-plane, $\left\langle h_{\perp}^{2}\right\rangle$, as well as out-of-plane, $\left\langle h_{\|}^{2}\right\rangle$, fields (only fluctuating field components perpendicular to the nuclear quantization axis lead to nuclear spin flips, required for spin-lattice relaxation).

Phonons will cause nuclear relaxation for quadrupolar nuclei $(I>1 / 2$, like $\mathrm{Cu}$ and $\mathrm{O})$ as they modulate the electric field gradient, but it has been shown that the magnetic fluctuations dominate in most situations [24, 25], and the recent analysis of all $\mathrm{Cu}$ relaxation data shows that a simple magnetic mechanism appears to capture the overall behavior quite well $[15,16]$.

In a straightforward approach, one would assume nearly isotropic spin fluctuations filtered by the nuclear hyperfine coefficients, which can then lead to a relaxation anisotropy. The electronic correlation time $\left(\tau_{0}\right)$ of electronic spin fluctuations is expected to be very fast compared with the slow precession of the nuclei. Thus, the nuclear relaxation rates can be written as [18]:

$$
\begin{aligned}
\frac{1}{T_{1 \|}} & =\frac{3}{2} \gamma^{2} \cdot 2\left\langle h_{\perp}^{2}\right\rangle \tau_{0} \\
\frac{1}{T_{1 \perp}} & =\frac{3}{2} \gamma^{2}\left[\left\langle h_{\perp}^{2}\right\rangle+\left\langle h_{\|}^{2}\right\rangle\right] \tau_{0},
\end{aligned}
$$

from which the relaxation anisotropy follows:

$$
\frac{1 / T_{1 \perp}}{1 / T_{1 \|}}=\frac{1}{2}+\frac{\left\langle h_{\|}^{2}\right\rangle}{2\left\langle h_{\perp}^{2}\right\rangle} .
$$

Given that the shifts demand two different electronic spin components coupled to the nuclei through an anisotropic constant $A_{\|, \perp}$ and an isotropic constant $B$, one should allow for two different fluctuating spin densities $\alpha$ and $\beta=\sum_{j} \beta_{j}$, as well. Furthermore, since the fluctuations are caused by rapid exchange, the correlation time $\tau_{0}$ should be the same for both components.

We thus write:

$$
\begin{aligned}
& \left\langle h_{\perp, \|}^{2}\right\rangle \approx\left\langle\left(\sum_{j} B \beta_{j}+A_{\perp, \|} \alpha\right)^{2}\right\rangle \\
& \left\langle h_{\|}^{2}\right\rangle \approx B^{2}\left\langle\left(\sum_{j} \beta_{j}-\alpha\right)^{2}\right\rangle, \\
& \left\langle h_{\perp}^{2}\right\rangle \approx B^{2}\left\langle\left(\sum_{j} \beta_{j}+f \alpha\right)^{2}\right\rangle,
\end{aligned}
$$

where we introduced $f=A_{\perp} / B \approx-A_{\perp} / A_{\|}$if $A_{\perp} \alpha$ is not negligible (see below).

With these expressions for the fluctuating field components, we seek to explain a rather doping- and materialindependent $1 / T_{1} \perp$ (it only increases marginally with decreasing doping) and a material- and doping-dependent $1 / T_{1 \|}$ that explain the temperature-independent anisotropy (14), as well as the exceptional behavior found for $\mathrm{La}_{2-x} \mathrm{Sr}_{x} \mathrm{CuO}_{4}$.

In the first scenario, one might be interested to see what would be the consequences of totally uncorrelated spin fluctuations for the 5 spin components, i.e., $\left\langle\beta_{i} \beta_{j}\right\rangle=$ $\left\langle\beta_{0}^{2}\right\rangle \delta_{i j}$, and $\left\langle\beta_{j} \alpha\right\rangle=0$, cf. Fig. 4. We then have $\left\langle h_{\perp}^{2}\right\rangle=$ $4\left\langle\beta_{0}^{2}\right\rangle$ and $\left\langle h_{\|}^{2}\right\rangle=4\left\langle\beta_{0}^{2}\right\rangle+\left\langle\alpha^{2}\right\rangle$, thus with (12) and (13) for uncorrelated $(u)$ fluctuations:

$$
\begin{aligned}
& \frac{1}{T_{1 \|, u}}=\frac{3}{2} \gamma^{2} B^{2} \cdot 8\left\langle\beta_{0}^{2}\right\rangle \tau_{0} \\
& \frac{1}{T_{1 \perp, u}}=\frac{3}{2} \gamma^{2} B^{2} \cdot\left[8\left\langle\beta_{0}^{2}\right\rangle+\left\langle\alpha^{2}\right\rangle\right] \tau_{0},
\end{aligned}
$$

and it follows for the anisotropy:

$\frac{1 / T_{1 \perp, u}}{1 / T_{1 \|, u}}=1+\frac{\left\langle\alpha^{2}\right\rangle}{8\left\langle\beta_{0}^{2}\right\rangle}$.

Clearly, for $\left\langle\alpha^{2}\right\rangle \lesssim\left\langle\beta_{0}^{2}\right\rangle$, we find near isotropic relaxation, and in order to explain the largest anisotropy of about 3.3 [16], we conclude $\left\langle\alpha^{2}\right\rangle \approx 18.4\left\langle\beta_{0}^{2}\right\rangle$. This implies, however, rather large changes of $\alpha$ and $\beta$ for meeting the experimental observations, i.e., the change in relaxation between materials and different doping levels, which appears to be difficult to meet in this approach (we do notice that a large $\alpha$ could be present, which demands that we do not neglect $A_{\perp}$ for the modeling of nuclear relaxation).

In the second scenario, cf. Fig. 4, we assume that all spins are aligned; i.e., the 5 fluctuating spin components are correlated, with $\left\langle\beta_{i} \beta_{j}\right\rangle=\left\langle\beta_{0}^{2}\right\rangle$ and $\left\langle\beta_{j} \alpha\right\rangle= \pm \alpha \beta_{0}$. We note 
Fig. 4 Fluctuating spins $\alpha$ and $\beta_{0}$, respectively located in the $\mathrm{Cu} 3 d\left(x^{2}-y^{2}\right)$ and $\mathrm{O} 2 p_{\sigma}$ orbital. a, all 5 spin components fluctuate independently, i.e., $\left\langle\alpha \beta_{0}\right\rangle=0,\left\langle\beta_{i} \beta_{j}\right\rangle=\beta_{0}^{2} \delta_{i j}$, $\mathbf{b}$, the fluctuations are fully correlated, i.e., $\left\langle\alpha \beta_{0}\right\rangle=\alpha \beta_{0},\left\langle\beta_{i} \beta_{j}\right\rangle=\beta_{0}^{2}$ a

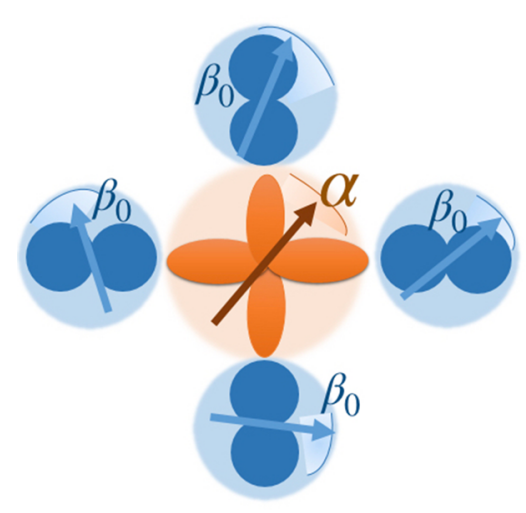

b

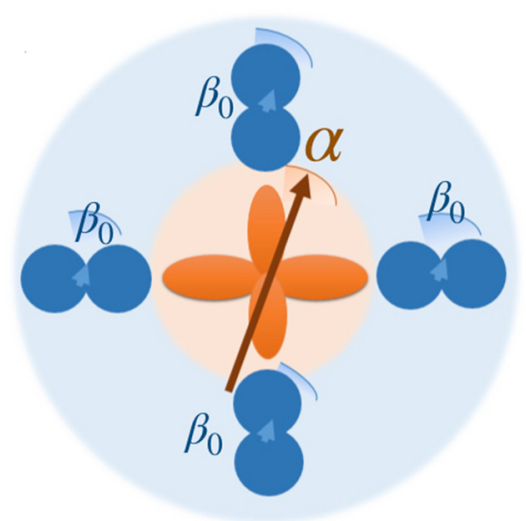

that the field fluctuations $\left\langle h_{\perp}^{2}\right\rangle \approx B^{2}\left\langle\left(\sum_{j} \beta_{j}+f \alpha\right)^{2}\right\rangle$ that enter (12) and $\left\langle h_{\|}^{2}\right\rangle \approx B^{2}\left\langle\left(\sum_{j} \beta_{j}-\alpha\right)^{2}\right\rangle$ that determine (13) are both quadratic in the resulting local spin densities. Therefore, in order to find a rather flat dependence for the relaxation for $c \perp B_{0}$ on $\beta_{0}$, as demanded by the experiment, we need to be close to its minimum, while at the same time, the parabola must be shifted by a negative $\alpha$ compared with the other parabola in order to meet a smaller but varying relaxation rate for $c \| B_{0}$. The results of simple calculations according to (12), (13) with (16) and (17) are shown in Fig. 5. We observe that there is only a special region with solutions that fit the experiments, for $\beta_{0} / \alpha=0.04$ to 0.11 according to anisotropies ranging from 3.3 to 1.0 , respectively, cf. Fig. 5.

Furthermore, an increase of $\alpha$ by a factor of about 1.3, at an anisotropy ratio of 2.3, increases the relaxation rates in both directions by about a factor of 2, cf. Fig. 5, which readily explains the data found for $\mathrm{La}_{2-x} \mathrm{Sr}_{x} \mathrm{CuO}_{4}$. We thus conclude that the two components $\beta_{0}$ and $\alpha$ are crucial for the cuprates, but appear to be very similar for most of the materials.

\section{Discussion}

It seems out of question that a two-component scenario describes the shifts and relaxation in the cuprates quite well. It has spin density located in the $\mathrm{Cu} 3 d\left(x^{2}-y^{2}\right)$ orbital, which couples to the nucleus through the rather anisotropic hyperfine constant $A_{\|, \perp}$, and, most likely, the planar O $2 p_{\sigma}$ orbital, leading to an isotropic hyperfine interaction given by (4).

The spin density $\alpha$ is much larger than $\beta_{0}$, as one expects from the overall material properties; however, the uniform response of both spins is quite different, also due to the coupling term $c_{j}$.

The special slopes observed in the shift-shift plot, cf. Fig. 1, are caused by changes of the individual spin components as a function of doping or temperature, except for the slope $\kappa \approx 2.5$ that must stem from a concomitant change of $b_{j}$ and $c_{j}$. This leads to the simple conclusion that $A_{\|} \approx-B$ (while $A_{\perp} \approx 0.15 A_{\|}$[23]), and it leaves us with a straightforward description of the spin shifts of the cuprates in terms of (10) and (11), i.e., $K_{\|} \approx B\left(4 b_{j}-a\right)$ and $K_{\perp} \approx B 4\left(b_{j}+c_{j}\right)$ (in these equations, we also adopted a different notation in terms of $b_{j}$ compared with our earlier analysis [15]). We note that the conclusion that $A_{\|} \approx-B$ has a similar origin as in the old interpretation.

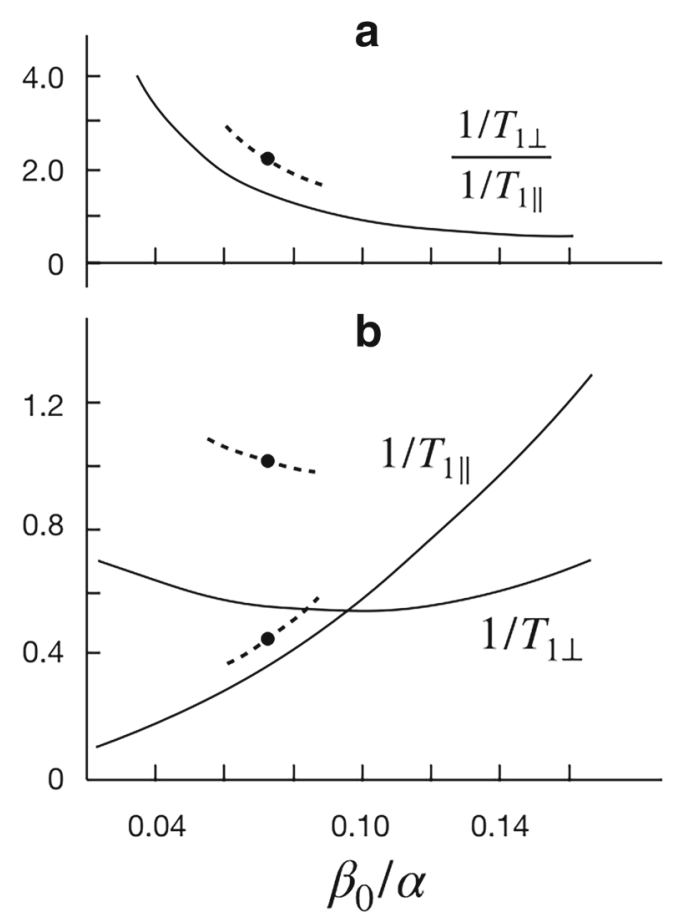

Fig. 5 a Calculated nuclear relaxation rates for $c \| B_{0}\left(1 / T_{1 \|}\right)$ and $c \perp B_{0}\left(1 / T_{1 \perp}\right)$ as a function of the ratio of the two spin components $\beta_{0} \equiv \beta_{j}$ and $\alpha\left(\beta_{0} / \alpha\right)$, according to (12), (13) with (16) and (17), in arbitrary units. $\mathbf{b}$ The anisotropy of the relaxation (14) varies between 4 and 0.5 in the same range of $\beta_{0} / \alpha$. Corresponding line segments for $\mathrm{La}_{2-x} \mathrm{Sr}_{x} \mathrm{CuO}_{4}$ with an anisotropy of about 2.3 are indicated, as well 
Looking again at Fig. 1b, the cuprates are sorted in this shift-shift plot effectively by the high-temperature $b_{j}$, the component that grows with increasing doping (toward the upper right in Fig. 1b). As the temperature is lowered, at a given temperature, which can be above or at $T_{\mathrm{c}}$, this term begins to disappear due to the action of $c_{j}$ (both components $c_{j}$ and $b_{j}$ can fall together, as well). It is the coupling to $a$ that sets $c_{j}$ (and effectively couples different $a$ terms, as well). The component $a$ appears to be temperature independent. It emerges that either $b_{j}$ or $c_{j}$ can be exhausted independently indicated by changes in slope at lower temperatures. However, all cuprates seem to reach the same $\left(b_{j}+c_{j}\right)=0$, which we define as zero spin shift. Importantly, there is no evidence that there is a different mechanism as one passes through $T_{\mathrm{c}}$ if the shift began to change already far above $T_{\mathrm{c}}$ (NMR pseudogap), but $c_{j}$ can traverse the region below $T_{\mathrm{c}}$ at a much higher rate for given steps in temperature.

The earlier conclusion $[14,15]$ that the spin shift for $c \| B_{0}$ does not disappear at low temperatures, here takes the formulation that $\left(4 b_{j}-a\right) \neq 0$ and says that the positive spin density $b_{j}$ and the negative spin density $a_{j}$ can remain temperature independent for systems with $\kappa \approx \infty$, or $b_{j}$ can also drop with $c_{j}$ as for the systems with slope $\kappa \approx 2.5$; i.e., it does not change in the condensed state, while relaxation ceases.

In terms of a simple fluctuating field model, we can explain the cuprate relaxation rather well. Fast electronic, Fermi liquid-like spin fluctuations act through two different hyperfine coefficients with two different electronic spin densities on the $\mathrm{Cu}$ nucleus (or, these densities are part of that ubiquitous fluid). The corresponding fluctuations from the 5 locations must be correlated, as one might have guessed due to the close proximity. The on-site $3 d\left(x^{2}-y^{2}\right)$ spin $(\alpha)$ is about 10 times as large as that due to one $\mathrm{O}$ neighbor $\left(\beta_{0}\right)$. The spin density $\alpha$ appears to be the same for all cuprates; except for the $\mathrm{La}_{2-x} \mathrm{Sr}_{x} \mathrm{CuO}_{4}$ family, it is $30 \%$ larger. The spin $\beta_{0}$ varies with doping and between materials and leads to the change in $1 / T_{1 \|}$ observed in the data. For large doping the relaxation anisotropy is about 1 and it increases to about 3.3 for $\mathrm{YBa}_{2} \mathrm{Cu}_{4} \mathrm{O}_{8}$ (corresponding to a change in $\beta_{0}$ of about 3). For $\mathrm{La}_{2-x} \mathrm{Sr}_{x} \mathrm{CuO}_{4}$ the anisotropy is 2.3 and thus also $\beta_{0}$ is about a factor of two larger.

Since the ${ }^{63} \mathrm{Cu}$ relaxation begins to disappear only at $T_{\mathrm{c}}$, for all cuprates, the electronic, Fermi liquid-like spin fluctuations freeze out and the relaxation disappears. Thus, the pseudogap in the relaxation is just due to the correlations that for planar $\mathrm{Cu}$ do not change $\alpha$ and $\beta_{0}$ spin alignment. For planar $\mathrm{O}$ the situation is different as the nucleus couples to two $\alpha$ spins at adjacent $\mathrm{Cu}$ nuclei and their coupling changes, which leads to the pseudogap in the relaxation for nuclei that are affected by different $a$ spins [26].
The relation between the spin densities $\alpha, \beta_{0}$ and the uniform response of the system in terms of $a, b_{j}$, and $c_{j}$ is not known. It appears that the response of $\alpha$ is rather small compared to that of $\beta$, which may not be surprising since different $a$ should favor antiferromagnetic alignment (that is somehow affected by $\beta$ ).

It appears that the doping dependent spread in $K_{\|}$varies among the cuprates. This reminds us of the way the charge carriers enter the $\mathrm{CuO}_{2}$ plane [12]. For the $\mathrm{La}_{2-x} \mathrm{Sr}_{x} \mathrm{CuO}_{4}$ family, the doped charges $x$ enter almost exclusively the $2 p_{\sigma}$ orbital $\left(n_{p}\right)$ while for other systems the $\mathrm{Cu} 3 d\left(x^{2}-y^{2}\right)$ $\left(n_{d}\right)$ is affected as well $\left(x=\Delta n_{d}+2 \Delta n_{p}[12,27]\right)$, and the spread in doping appears to grow with $\Delta n_{d}$. The maximum achievable $T_{\mathrm{c}}$, however, is set by the sharing of the parent material's hole content, i.e., $n_{d}^{*}+2 n_{p}^{*}=1$ and $T_{\mathrm{c}, \max } \propto n_{p}^{*}[12,13,28]$. Materials with the highest $T_{\mathrm{c}}$ appear to adopt $\kappa \approx 2.5$, only. However, the jumping between different slopes $\kappa$ in different regions of the shiftshift plot that involves $b_{j}$ and/or $c_{j}$ below $T_{\mathrm{c}}$ is absent for optimally doped systems, which probably means that $b_{j}$ and $c_{j}$ are matched at optimal doping.

Finally, one may argue that the intra cell charge variation between neighboring planar $\mathrm{O}$ atoms that appears to be ubiquitous and that can respond to the external magnetic field $[22,29]$ could be involved in the two component scenario.

\section{Conclusions}

Two spin densities were shown to reside in the planar $\mathrm{Cu} 3 d\left(x^{2}-y^{2}\right)$ and likely the planar $\mathrm{O} 2 p_{\sigma}$ orbitals, respectively, with hyperfine constants $A_{\|, \perp}$ and $B \approx$ $-A_{\|}$. They connect the $\mathrm{Cu}$ nuclear spins with a rather ubiquitous Fermi liquid-like bath. The relaxation anisotropy is predominantly due to changes in the planar $\mathrm{O}$ spin density that increases with doping. Near $T_{\mathrm{c}}$, these electronic fluctuations freeze out and the relaxation disappears.

The uniform response $a$ and $b_{j}$ of the two electronic spins on $\mathrm{Cu}$ and $\mathrm{O}$ is special in the sense that $a$ is negative while $b_{j}$ points along the field. The coupling term $c_{j}$ between $a$ and $b_{j}$ sets the temperature dependence of the shift above (NMR pseudogap) and below $T_{\mathrm{c}}$. Interestingly, at the lowest temperatures, $4\left(b_{j}+c_{j}\right)$ approaches the same value for all cuprates, probably zero, but $a$ remains and most of $b_{j}$, as well, resulting in a non-vanishing spin shift for $c \| B_{0}$, $K_{\|} \approx\left(4 b_{j}-a\right) \neq 0$.

The coupling term $c_{j}$ must be related to a coupling between different spin components $a_{i}$ on different $\mathrm{Cu}$ nuclei, and it is argued that the pseudogap phenomenon for planar $\mathrm{O}$ nuclear relaxation, and that of $\mathrm{Y}$, is just a consequence of the temperature dependence of $c_{j}$, an effect that cannot be there in the $\mathrm{Cu}$ relaxation data. 
This simple two-component scenario appears to fit all cuprates, in particular also the only outlier family so far, $\mathrm{La}_{2-x} \mathrm{Sr}_{x} \mathrm{CuO}_{4}$, which must make it a reliable framework for theory.

Acknowledgments We acknowledge support from Leipzig University, and fruitful discussions with A. Poeppl, M. Jurkutat, and A. Kreisel.

Author Contributions J.H. introduced the main concepts and held the overall leadership; all authors were involved in data analysis and discussion equally, as well as in preparing the manuscript.

Funding Information Open Access funding provided by Projekt DEAL.

Open Access This article is licensed under a Creative Commons Attribution 4.0 International License, which permits use, sharing, adaptation, distribution and reproduction in any medium or format, as long as you give appropriate credit to the original author(s) and the source, provide a link to the Creative Commons licence, and indicate if changes were made. The images or other third party material in this article are included in the article's Creative Commons licence, unless indicated otherwise in a credit line to the material. If material is not included in the article's Creative Commons licence and your intended use is not permitted by statutory regulation or exceeds the permitted use, you will need to obtain permission directly from the copyright holder. To view a copy of this licence, visit http:// creativecommonshorg/licenses/by/4.0/.

\section{References}

1. Slichter, C.P. Principles of Magnetic Resonance, 3rd edn. Springer, Berlin (1990)

2. Hebel, L.C., Slichter, C.P.: Phys. Rev. 113, 1504 (1959). https://doi.org/10.1103/PhysRev.113.1504

3. Yosida, K.: Phys. Rev. 110, 769 (1958). https://doi.org/10.1103/ PhysRev.110.769

4. Bednorz, J.G., Müller, K.A., Phys, Z., Condens, B.: Matter 193, 189 (1986). https://doi.org/10.1007/BF01303701

5. Slichter, C.P.: In: Schrieffer, J.R., Brooks, J.S. (eds.) Handbook of High-Temperature Superconductivity, pp. 215-256. Springer, New York (2007). https://doi.org/10.1007/978-0-387-68734-6_5

6. Walstedt, R.E.: The NMR Probe of High- $\mathrm{T}_{\mathrm{c}}$ Materials, $1 \mathrm{st}$ edn, Springer. https://doi.org/10.1007/978-3-540-75565-4 (2007)

7. Haase, J., Slichter, C.P., Williams, G.V.M.: J. Phys. Condens. Matter 21, 455702 (2009). https://doi.org/10.1088/0953-8984/21/ $45 / 455702$

8. Meissner, T., Goh, S.K., Haase, J., Williams, G.V.M., Littlewood, P.B.: Phys. Rev. B 83, 220517 (2011). https://doi.org/10.1103/ PhysRevB.83.220517
9. Haase, J., Rybicki, D., Slichter, C.P., Greven, M., Yu, G., Li, Y., Zhao, X.: Phys. Rev. B 85, 104517 (2012). https://doi.org/10. 1103/PhysRevB.85.104517

10. Rybicki, D., Kohlrautz, J., Haase, J., Greven, M., Zhao, X., Chan, M.K., Dorow, C.J., Veit, M.J.: Phys. Rev. B 92, 081115 (2015). https://doi.org/10.1103/PhysRevB.92.081115

11. Zheng, G.Q., Mito, T., Kitaoka, Y., Asayama, K., Kodama, Y.: Phys. C Supercond. 243, 337 (1995). https://doi.org/10.1016/ 0921-4534(95)00029-1

12. Jurkutat, M., Rybicki, D., Sushkov, O.P., Williams, G.V.M., Erb, A., Haase, J.: Phys. Rev. B 90, 140504 (2014). https://doi.org/10. 1103/PhysRevB.90.140504

13. Rybicki, D., Jurkutat, M., Reichardt, S., Kapusta, C., Haase, J.: Nat. Commun. 7, 1 (2016). https://doi.org/10.1038/ncomms11413

14. Haase, J., Jurkutat, M., Kohlrautz, J.: Condens. Matter 2(2), 16 (2017)

15. Avramovska, M., Pavićević, D., Haase, J.: J. Supercond Nov. Magn. 243(3), 337 (2019)

16. Jurkutat, M., Avramovska, M., Williams, G.V.M., Dernbach, D., Pavićević, D., Haase, J.: J. Supercond Nov. Magn. 155(12), 629 (2019)

17. Ohsugi, S., Kitaoka, Y., Ishida, K., Aheng, G.Q., Asayama, K.: J. Phys. Soc. Jpn. 63, 700 (1994). https://doi.org/10.1143/JPSJ. 63.700

18. Pennington, C.H., Durand, D.J., Slichter, C.P., Rice, J.P., Bukowski, E.D., Ginsberg, D.M.: Phys. Rev. B 39, 2902 (1989). https://doi.org/10.1103/PhysRevB.39.2902

19. Renold, S., Heine, T., Weber, J., Meier, P.F.: Phys. Rev. B 67(2), 24501 (2003). https://doi.org/10.1103/PhysRevB.67.024501

20. Pavićević, D., Avramovska, M., Haase, J.: arXiv.org (2019)

21. Haase, J.: Phys. Rev. Lett. 91(18), 189701 (2003)

22. Korringa, J.: Physica 16, 601 (1950). https://doi.org/10.1016/ 0031-8914(50)90105-4

23. Husser, P., Suter, H.U., Stoll, E.P., Meier, P.F.: Phys. Rev. B 61(2), $1567(2000)$

24. Takigawa, M., Smith, J.L., Hults, W.L.: Phys. Rev. B 44(14), 7764 (1991)

25. Suter, A., Mali, M., Roos, J., Brinkmann, D.: J. Magn. Reson. 143(2), 266 (2000)

26. Avramovska, M., Pavićević, D., Haase, J. ((in preparation))

27. Haase, J., Sushkov, O.P., Horsch, P., Williams, G.V.M.: Phys. Rev. B 69, 94504 (2004). https://doi.org/10.1103/PhysRevB.69.094504

28. Jurkutat, M., Erb, A., Haase, J.: Condens. Matter 4(3), 67 (2019)

29. Reichardt, S., Jurkutat, M., Guehne, R., Kohlrautz, J., Erb, A., Haase, J.: Condens. Matter 3(3), 23 (2018)

Publisher's Note Springer Nature remains neutral with regard to jurisdictional claims in published maps and institutional affiliations. 